\title{
ON A HIGH ORDER NUMERICAL METHOD FOR FUNCTIONS WITH SINGULARITIES
}

\author{
KNUT S. ECKHOFF
}

\begin{abstract}
By splitting a given singular function into a relatively smooth part and a specially structured singular part, it is shown how the traditional Fourier method can be modified to give numerical methods of high order for calculating derivatives and integrals. Singular functions with various types of singularities of importance in applications are considered. Relations between the discrete and the continuous Fourier series for the singular functions are established. Of particular interest are piecewise smooth functions, for which various important applications are indicated, and for which numerous numerical results are presented.
\end{abstract}

\section{INTRODUCTION}

For various applications we may be concerned with functions $w(x)$ given on the interval $[0,2 \pi]$, say, under circumstances where it is known that the otherwise smooth function $w(x)$ has special features (e.g. singularities) at a finite number of points $x=\gamma_{j}, j=1,2, \ldots, M$, say. Of particular interest here are piecewise smooth functions which occur for instance in problems with shocks [9], [12], [13], but also in other important applications [8], [10], [11], [14]. For a piecewise smooth function $w(x)$ we have in these earlier works found it advantageous for a given integer $Q \geq 0$ to write $w(x)$ in the following way on $[0,2 \pi]$

$$
w(x)=w^{Q}(x)+\sum_{n=0}^{Q} \sum_{j=1}^{M} A_{j}^{n} U_{n}\left(x-\gamma_{j}\right) .
$$

For each $n=0,1,2, \ldots$, the $2 \pi$-periodic function $U_{n}(x)$ is here a piecewise polynomial of degree $n+1$ given by

$$
U_{n}(x)=-\frac{(2 \pi)^{n}}{(n+1) !} B_{n+1}\left(\frac{x}{2 \pi}\right) \quad \text { when } 0<x<2 \pi,
$$

where $B_{j}(x), j=1,2, \ldots$, are the Bernoulli polynomials, [10], [15]. The representation (1) is a generalization of a representation utilized by Lanczos [20] for functions with one singularity in each period (i.e. $M=1$ and $\gamma_{1}=0$ ), but apparently [18] the

Received by the editor December 11, 1996 and, in revised form, March 26, 1997.

1991 Mathematics Subject Classification. Primary 65M70, 65N35.

Key words and phrases. Spectral methods, Fourier series, discontinuous functions, Bernoulli polynomials, singular functions, quadrature, partial differential equations.

This paper is partly based on work done while the author was engaged at the SINTEF Multiphase Flow Laboratory, Trondheim, Norway. The paper is also partly based on work done while the author was in residence at the Division of Applied Mathematics, Brown University, Providence, R.I., U.S.A. supported by AFOSR grant 95-1-0074 and NSF grant DMS-9500814.

(C)1998 American Mathematical Society 
basic idea was introduced earlier by A. N. Krylov. From (2) it follows that $U_{n}(x)$ is $n-1$ times continuously differentiable everywhere, while the $n$th derivative of $U_{n}(x)$ suffers a jump discontinuity of magnitude +1 at $x=0, \pm 2 \pi, \pm 4 \pi, \ldots$. Hence, if for each $n=0,1,2, \ldots, Q$ we let $A_{j}^{n}$ be given as the jump in the $n^{\prime}$ th derivative of the function $w(x)$ at the singularity location $x=\gamma_{j}$

$$
A_{j}^{n}=\frac{d^{n} w}{d x^{n}}\left(\gamma_{j}^{+}\right)-\frac{d^{n} w}{d x^{n}}\left(\gamma_{j}^{-}\right)
$$

and if we include the point $x=0$ among the singularity locations $\gamma_{j}$ whenever that is necessary [11], it readily follows from (1) that the $2 \pi$-periodic extension of the function $w^{Q}(x)$ is continuous and $Q$ times continuously differentiable everywhere. We furthermore note that the Fourier coefficients associated with the function $U_{n}(x)$ are given by

$$
{\widehat{\left(U_{n}\right)_{0}}}_{0}=0,{\widehat{\left(U_{n}\right)_{k}}}_{k}=\frac{1}{2 \pi(i k)^{n+1}} ; k= \pm 1, \pm 2, \ldots
$$

In this paper we shall utilize the representation (1) further for piecewise smooth functions $w(x)$, and in particular consider new ways of determining the jumps (3) when they are not known in advance. We note here that the lack of robust methods for jump-determination has been the main reason why this type of construction has not been extensively utilized earlier [21, p.101]. It may also seem natural to try to generalize the representation (1) to cover functions $w(x)$ with other types of singularities than jump-singularities. Thus we shall consider the more general representation

$$
w(x)=w^{Q}(x)+\sum_{n=0}^{Q} \sum_{j=1}^{M} A_{j}^{n} V_{n}\left(x ; \gamma_{j}\right),
$$

where the functions $V_{n}\left(x ; \gamma_{j}\right)$ are assumed to possess prototype special features (singularities) of the same kind that $w(x)$ is known to have at the point $x=\gamma_{j}$ in such a way that for certain constants $A_{j}^{n}$ (i.e. strengths of the singularities), the function $w^{Q}(x)$ in (5) becomes less singular the higher we choose the value of $Q$.

The assumed existence of the special feature functions $V_{n}\left(x ; \gamma_{j}\right)$ is crucial for the theory we are going to develop in this paper. In addition to the above properties, further properties will be specified in section 4 for these functions. We do not attempt here to give a theory for how the functions $V_{n}\left(x ; \gamma_{j}\right)$ can be chosen in general, but in section 3 we shall study in some detail a relatively large family of functions from which it is possible to choose the special feature functions $V_{n}\left(x ; \gamma_{j}\right)$ in many important special cases. For various other cases, we suggest that asymptotic expansions near the singularities [3] may be a useful tool for the construction of the relevant special feature functions $V_{n}\left(x ; \gamma_{j}\right)$.

The primary purpose of the representation (5) in our context is its suitability for calculating integrals or derivatives of the given singular function $w(x)$. When $x \neq \gamma_{1}, \ldots, \gamma_{M}$, differentiation of (5) clearly leads to

$$
\frac{d^{m}}{d x^{m}} w(x)=\frac{d^{m}}{d x^{m}} w^{Q}(x)+\sum_{n=0}^{Q} \sum_{j=1}^{M} A_{j}^{n} \frac{d^{m}}{d x^{m}} V_{n}\left(x ; \gamma_{j}\right) .
$$

From this equation and the fact that the function $w^{Q}(x)$ is relatively smooth, we may conclude that derivatives of the singular function $w(x)$ can be accurately calculated by standard methods if the special feature functions $V_{n}\left(x ; \gamma_{j}\right)$ are explicitly 
known and the quantities $A_{j}^{n}$ in (5) can be accurately determined for $n=0,1, \ldots, Q$ and $j=1, \ldots, M$. Analogous remarks hold for integrals of the singular function $w(x)$.

Thus, an important purpose of the present paper is to provide efficient, accurate and robust methods for determining the quantities $A_{j}^{n}$ in (5) when the relevant special feature functions $V_{n}\left(x ; \gamma_{j}\right)$ have been found. Utilizing properties established in section 4 , this will be done in section 5 . Fourier methods will provide the basic tools for our constructions, we shall therefore in section 2 describe the fundamental results needed from Fourier analysis. In section 6 we shall briefly discuss how the methods described in this paper can be efficiently utilized as tools both for numerical differentiation as well as for numerical integration in the one-dimensional as well as in the multi-dimensional case. In section 7 we shall give some numerical results obtained by the described approach, and, finally, in section 8 we shall give some concluding remarks.

\section{THE Fourier METHOD}

To an integrable complex-valued $2 \pi$-periodic function $u(x)$ we may for any given integer $N>0$ associate the $N$ th-order truncated Fourier series

$$
P_{N} u(x)=\sum_{k=-N}^{N} \hat{u}_{k} e^{i k x},
$$

where

$$
\hat{u}_{k}=\frac{1}{2 \pi} \int_{0}^{2 \pi} u(x) e^{-i k x} d x ; \quad k=0, \pm 1, \pm 2, \ldots
$$

An extensive theory for Fourier series is established in the literature [6], [17], [24]. The error involved when we approximate $u(x)$ by the truncated Fourier series expansion (7) is known to be strongly dependent on the smoothness of the function $u(x)$. By introducing notations which differs slightly from the standard ones, it will be shown in this section that all the results we shall need from Fourier analysis can be established by elementary proofs only. The following class of functions will be seen to constitute a suitable basis for our theory.

Definition 1. $S_{2 \pi}^{\alpha}$ is the class of all $2 \pi$-periodic integrable functions $u(x)$ which are such that $\sum_{k=-\infty}^{+\infty}(|k|+1)^{\beta}\left|\hat{u}_{k}\right|$ converges for every $\beta<\alpha$.

From this definition it readily follows that $S_{2 \pi}^{\alpha} \subset S_{2 \pi}^{\gamma}$ whenever $\alpha>\gamma$. If $u \in S_{2 \pi}^{\alpha}$ for some $\alpha>0$, its infinite Fourier series $\lim _{N \rightarrow \infty} P_{N} u(x)$ is seen to be absolutely and uniformly convergent, and $u(x)$ is consequently a.e. equal to a continuous function. Similarly, we see that if $u \in S_{2 \pi}^{\alpha}$ for some $\alpha>m, u(x)$ is a.e. equal to a continuous function which is $m$ times continuously differentiable. Since we shall not distinguish between ordinary functions which are different only on a set of measure zero, we therefore have that $S_{2 \pi}^{\alpha} \subset C^{m}$ whenever $\alpha>m$. By utilizing the Schwarz inequality, it is furthermore easily seen that the Sobolev space of $2 \pi$ periodic functions $H^{s} \subset S_{2 \pi}^{s-\frac{1}{2}}$. When $u^{\prime}$ denotes the derivative in the sense of distributions, we see that $u^{\prime} \in S_{2 \pi}^{\alpha-1}$ whenever $u \in S_{2 \pi}^{\alpha}$. This can be used to extend the above definition to also cover certain non-integrable functions as well as generalized functions. 
From the above definition it clearly follows that if we have

$$
\hat{u}_{k}=O\left(|k|^{-\alpha-1}\right) \text { as } k \rightarrow \pm \infty,
$$

then $u \in S_{2 \pi}^{\alpha}$. If in particular we assume that the $2 \pi$-periodic function $u(x)$ is continuous and $m-1$ times continuously differentiable everywhere, and that $u^{(m)}(x)$ is piecewise continuous and piecewise differentiable on $[0,2 \pi]$, it can be readily shown by partial integration and Riemann-Lebesgue's Lemma that

$$
\hat{u}_{k}=O\left(|k|^{-m-1}\right) \quad \text { as } \quad k \rightarrow \pm \infty .
$$

Thus it follows that in this case $u \in S_{2 \pi}^{m}$. If $u(x)$ is discontinuous, but such that $u(x)$ is piecewise continuous and piecewise differentiable on $[0,2 \pi]$, then (10) holds with $m=0$ and $u \in S_{2 \pi}^{0}$. It should be emphasized, however, that (9) does not hold for all functions $u \in S_{2 \pi}^{\alpha}$, and in particular that the piecewise smooth functions only constitute a small subset of the class of functions $S_{2 \pi}^{0}$.

In order to establish suitable estimates, it will be convenient in the following to apply the following notation

Definition 2. A sequence $\left\{g_{k}\right\}$ is said to be of the order $O_{s}\left(k^{-\alpha}\right)$ as $k \rightarrow \infty$, if for every $\beta<\alpha$ we have $g_{k}=o\left(k^{-\beta}\right)$ as $k \rightarrow \infty$.

It is easy to construct examples showing that $O_{s}\left(k^{-\alpha}\right)$ does not in general imply the slightly sharper estimate $O\left(k^{-\alpha}\right)$ as $k \rightarrow \infty$. Although formally not equivalent, it seems difficult in practice to distinguish between those two estimates in actual numerical computations, however. As an immediate application of the notation introduced in Definition 2, and as a contrast to the sufficient condition (9), it easily follows from Definition 1 that a necessary condition for $u \in S_{2 \pi}^{\alpha}$ is that

$$
\hat{u}_{k}=O_{s}\left(|k|^{-\alpha}\right) \quad \text { as } \quad k \rightarrow \pm \infty .
$$

When the function $u(x)$ and its derivatives up to the order $p$ at every point are given by the corresponding infinite Fourier series (a sufficient condition is that $u \in S_{2 \pi}^{\alpha}$ for some $\alpha>p$ ), it follows for the truncation-error

$$
E_{N} u(x) \stackrel{\text { def }}{=} u(x)-P_{N} u(x)=\sum_{|k|=N+1}^{+\infty} \hat{u}_{k} e^{i k x},
$$

that for arbitrary $\gamma \geq 0$ we have

$$
\left\|\frac{d^{p} E_{N} u(x)}{d x^{p}}\right\| \stackrel{\text { def }}{=} \max _{0 \leq x \leq 2 \pi}\left|\frac{d^{p} E_{N} u(x)}{d x^{p}}\right| \leq N^{-\gamma} \sum_{|k|=N+1}^{+\infty}(|k|+1)^{p+\gamma}\left|\hat{u}_{k}\right| .
$$

Assuming that $\gamma$ is such that the sum on the right hand side in (13) converges, we may at least for $u \in S_{2 \pi}^{\alpha}$ with $\alpha>p$ conclude that

$$
\left\|\frac{d^{p} E_{N} u(x)}{d x^{p}}\right\|=O_{s}\left(N^{p-\alpha}\right) \quad \text { as } \quad N \rightarrow \infty .
$$

If $u \in S_{2 \pi}^{m}$ is such that $u^{(m)}(x)$ is piecewise continuous and piecewise differentiable on $[0,2 \pi]$, it can be shown that the following slightly sharper estimate holds when $m \geq p$

$$
\left\|\frac{d^{p} E_{N} u(x)}{d x^{p}}\right\|=O\left(N^{p-m}\right) \quad \text { as } \quad N \rightarrow \infty .
$$


Let us now assume that the function $u(x)$ is known (and finite) on a uniform set of, say, $G$ grid points in the interval $[0,2 \pi]$

$$
x_{l}=\frac{2 \pi}{G} l ; \quad l=0,1, \ldots, G-1 .
$$

The discrete Fourier coefficients associated with $u(x)$ are then as usual given by [6]

$$
\tilde{u}_{k} \stackrel{\text { def }}{=} \frac{1}{G} \sum_{l=0}^{G-1} u\left(x_{l}\right) e^{-i k x_{l}}, \quad k=0, \pm 1, \pm 2, \ldots, \pm\left[\frac{G-1}{2}\right] .
$$

Assuming that either $G=2 N+1$ or $G=2(N+1)$, we define the interpolated Fourier series of order $N$ associated with $u(x)$ by

$$
I_{N} u(x) \stackrel{\text { def }}{=} \sum_{k=-N}^{N} \tilde{u}_{k} e^{i k x} .
$$

Strictly speaking, (18) is interpolating the given values of the function $u(x)$ at the collocation points (16) only when $G=2 N+1$. We shall use the form (18) also for $G=2(N+1)$, however, in order to avoid the nuisance introduced by the unsymmetric series which exactly interpolates the given values of $u(x)$ at an even number of collocation points (16) [6].

When $u \in S_{2 \pi}^{\alpha}$ for some $\alpha>0$, the following well-known relation [6]

$$
\tilde{u}_{k}=\hat{u}_{k}+\sum_{m=1}^{+\infty}\left[\hat{u}_{k+m G}+\hat{u}_{k-m G}\right],
$$

can be readily utilized to establish the estimate

$$
\tilde{u}_{k}=\hat{u}_{k}+O_{s}\left(N^{-\alpha}\right) \text { as } N \rightarrow \infty .
$$

For the interpolation-error, i.e. the difference between the truncated Fourier series (7) and the interpolated series (18), we clearly have by (19) for $\beta \geq 0$

$$
\begin{gathered}
\left\|\frac{d^{p} P_{N} u(x)}{d x^{p}}-\frac{d^{p} I_{N} u(x)}{d x^{p}}\right\|=\left\|\sum_{k=-N}^{N}(i k)^{p} \sum_{m=1}^{+\infty}\left[\hat{u}_{k+m G}+\hat{u}_{k-m G}\right] e^{i k x}\right\| \\
\leq N^{p} \sum_{k=-N}^{N} \sum_{m=1}^{+\infty}\left[\left|\hat{u}_{k+m G}\right|+\left|\hat{u}_{k-m G}\right|\right] \leq N^{p-\beta} \sum_{|k|=N+1}^{+\infty}(|k|+1)^{\beta}\left|\hat{u}_{k}\right| .
\end{gathered}
$$

When $u \in S_{2 \pi}^{\alpha}$ with $\alpha>p$, we may therefore conclude from (21) that

$$
\left\|\frac{d^{p} P_{N} u(x)}{d x^{p}}-\frac{d^{p} I_{N} u(x)}{d x^{p}}\right\|=O_{s}\left(N^{p-\alpha}\right) \quad \text { as } \quad N \rightarrow \infty .
$$

The interpolation-error (22) is thus seen to be of exactly the same order as the truncation error (14) [5], [19].

\section{Special Families of FUnCTIONS}

Let $\alpha$ be a real number and let us consider the family of $2 \pi$-periodic functions $f_{\alpha}(x)$ given by

$$
f_{\alpha}(x)=\frac{x^{\alpha}}{\Gamma(\alpha+1)} \quad \text { when } \quad 0<x<2 \pi
$$


The function $f_{\alpha}(x)$ is clearly integrable if and only if $\alpha>-1$, and the associated Fourier coefficients (8) are given by

$$
\widehat{\left(f_{\alpha}\right)_{k}}=\frac{1}{2 \pi} \int_{0}^{2 \pi} \frac{x^{\alpha} e^{-i k x}}{\Gamma(\alpha+1)} d x ; \quad k=0, \pm 1, \pm 2, \ldots
$$

In particular, we therefore have

$$
\widehat{\left(f_{\alpha}\right)_{0}}=\frac{(2 \pi)^{\alpha}}{\Gamma(\alpha+2)}
$$

In order to get hold of the other coefficients (24) when $\alpha$ is not an integer, we denote by $m \geq 0$ the integer which is such that $m-1<\alpha<m$. By applying partial integration $m$ times in (24), we may then rewrite the result in the following way [3] when $m \geq 1$

$$
\begin{gathered}
\widehat{\left(f_{\alpha}\right)_{k}}=\frac{1}{2 \pi(i k)^{m}} \int_{0}^{2 \pi} \frac{x^{\alpha-m} e^{-i k x}}{\Gamma(\alpha+1-m)} d x-\sum_{n=1}^{m} \frac{(2 \pi)^{\alpha-n}}{(i k)^{n} \Gamma(\alpha+2-n)} \\
=\frac{1}{2 \pi(i k)^{m}} \int_{0}^{+\infty} \frac{x^{\alpha-m} e^{-i k x}}{\Gamma(\alpha+1-m)} d x-\frac{1}{2 \pi(i k)^{m}} \int_{2 \pi}^{+\infty} \frac{x^{\alpha-m} e^{-i k x}}{\Gamma(\alpha+1-m)} d x \\
-\sum_{n=1}^{m} \frac{(2 \pi)^{\alpha-n}}{(i k)^{n} \Gamma(\alpha+2-n)} .
\end{gathered}
$$

Since $-1<\alpha-m<0$, it is not difficult to show that [3, p. 311]

$$
\frac{1}{2 \pi(i k)^{m}} \int_{0}^{+\infty} \frac{x^{\alpha-m} e^{-i k x}}{\Gamma(\alpha+1-m)} d x=\frac{1}{2 \pi(i k)^{\alpha+1}} .
$$

Now let $Q>\alpha-1$ be an arbitrarily given non-negative integer. Application of (27) and partial integration $Q+1-m$ times in the last integral in (26) then give us asymptotically as $k \rightarrow \pm \infty$

$$
\begin{gathered}
\widehat{\left(f_{\alpha}\right)_{k}}=\frac{1}{2 \pi(i k)^{\alpha+1}}-\sum_{n=0}^{Q} \frac{(2 \pi)^{\alpha-n-1}}{(i k)^{n+1} \Gamma(\alpha+1-n)}+O\left(|k|^{-Q-2}\right) \\
=\frac{1}{2 \pi(i k)^{\alpha+1}}-\sum_{n=0}^{Q} \frac{(2 \pi)^{\alpha-n}}{\Gamma(\alpha+1-n)}{\widehat{\left(U_{n}\right)_{k}}}+O\left(|k|^{-Q-2}\right),
\end{gathered}
$$

where the Fourier coefficients (4) have been employed. From (9), (28), we may conclude that $f_{\alpha} \in S_{2 \pi}^{\alpha}$ when $-1<\alpha<0$, while $f_{\alpha} \in S_{2 \pi}^{0}$ when $\alpha>0$.

With reference to the first term on the right hand side in (28), we now let $U_{\alpha}(x) \in S_{2 \pi}^{\alpha}$ denote the $2 \pi$-periodic function which has the Fourier coefficients $\widehat{\left(U_{\alpha}\right)_{0}}=0$ and

$$
{\widehat{\left(U_{\alpha}\right)_{k}}}_{k}=\frac{1}{2 \pi(i k)^{\alpha+1}}=\frac{e^{-i \frac{\pi}{2}(\alpha+1) \operatorname{sign} k}}{2 \pi|k|^{\alpha+1}} ; k= \pm 1, \pm 2, \ldots .
$$

If $\alpha=n$ is a non-negative integer, these Fourier coefficients (29) coincide with the coefficients (4). Hence the family of functions $U_{\alpha}(x)$ is a generalization of the family of functions $U_{n}(x)$ given by (2) to non-integer values of the parameter $\alpha$. 
With (29), the Fourier series associated to $U_{\alpha}(x)$ can be written

$$
U_{\alpha}(x) \sim \frac{1}{\pi} \sum_{k=1}^{\infty}\left(\frac{1}{k}\right)^{\alpha+1} \cos \left[\frac{\pi}{2}(\alpha+1)-k x\right] .
$$

For $\alpha>0$, the series (30) is clearly absolutely and uniformly convergent everywhere. For $\alpha=0$, the series (30) converges everywhere, but no longer uniformly in a neighborhood of $x=0, \pm 2 \pi, \pm 4 \pi, \ldots$. For $-1<\alpha<0$, the magnitudes of the coefficients in (30) are still monotonically decreasing as $|k| \rightarrow \infty$, hence (30) is known [24] to converge everywhere with the exception of the single point $x=$ $0, \pm 2 \pi, \pm 4 \pi, \ldots$ in each period.

With the exception of the leading $(k=1)$ Fourier coefficient in (30), all coefficients are seen to be monotonically decreasing when $\alpha$ increases. Since the rate of decrease is seen to be increasing with the value of $k$, the associated truncated Fourier series (7) will accurately approximate $U_{\alpha}(x)$ for moderate or small values of $N$ if $\alpha$ is chosen sufficiently large. In fact, for $\alpha \geq 50, U_{\alpha}(x)$ can be approximated with machine accuracy for normal double precision calculations by the leading term in $(30)$

$$
U_{\alpha}(x) \approx \frac{1}{\pi} \cos \left[\frac{\pi}{2}(\alpha+1)-x\right] .
$$

The approximation (31) is fairly good also for smaller values of $\alpha$ than 50, but the error increases as $\alpha$ decreases. For $\alpha=6$, for instance, the error for the approximation (31) is everywhere less than $2.6 \cdot 10^{-3}$. In order to approximate $U_{\alpha}(x)$ with machine accuracy, it suffices to keep 10 terms in (30) for $\alpha \geq 15,20$ terms for $\alpha \geq 11$, while in our calculations we needed 456 terms for $\alpha=5$ and 1555 terms for $\alpha=4$ in order to get the optimal approximation for normal double precision calculations (53 bits). For $\alpha$ smaller than 4 , the truncated Fourier series soon becomes impractical for obtaining machine accuracy, since the necessary number of terms will be sky-rocketing beyond any limit of practical interest the closer we get to the value $\alpha=-1$, where the Fourier series no longer converges.

In later applications we shall need accurate information about the function $U_{\alpha}(x)$ for $\alpha$ small, i.e. for cases where the associated Fourier series (30) is converging very slowly. In fact, we are for some applications interested in information not only about the function $U_{\alpha}(x)$ itself, but also about its derivatives when $-1<\alpha<0$. In order to obtain the necessary information, we may write $U_{\alpha}(x)$ in the following way

$$
U_{\alpha}(x)=f_{\alpha}(x)+\Phi_{\alpha}^{Q}(x)+\Psi_{\alpha}^{Q}(x) .
$$

Here $f_{\alpha}(x)$ is the $2 \pi$-periodic function given by $(23)$ and $\Psi_{\alpha}^{Q}(x)$ is the following explicit linear combination of the $2 \pi$-periodic functions $U_{n}(x)$ given by $(2)$

$$
\Psi_{\alpha}^{Q}(x) \stackrel{\text { def }}{=} \sum_{n=0}^{Q} C_{\alpha}^{n} U_{n}(x) \quad \text { where } \quad C_{\alpha}^{n}=\frac{(2 \pi)^{\alpha-n}}{\Gamma(\alpha+1-n)},
$$

while $\Phi_{\alpha}^{Q}(x)$ is a $2 \pi$-periodic function which by (28) is seen to satisfy

$$
\widehat{\left(\Phi_{\alpha}^{Q}\right)_{k}}=O\left(|k|^{-Q-2}\right) \quad \text { as } k \rightarrow \pm \infty .
$$

Hence $\Phi_{\alpha}^{Q} \in S_{2 \pi}^{Q+1}$ by (9). With this background we may conclude that $U_{\alpha}(x)$ is smooth everywhere in the open interval $(0,2 \pi)$ and furthermore that $U_{\alpha}(x)-g_{\alpha}(x)$ 
is smooth in a neighborhood of $x=0$ when we choose

$$
g_{\alpha}(x)= \begin{cases}0 & \text { when } x<0 \\ f_{\alpha}(x) & \text { when } x>0\end{cases}
$$

Thus (35) characterizes the type of singularity the otherwise smooth function $U_{\alpha}(x)$ has at $x=0, \pm 2 \pi, \pm 4 \pi, \ldots$. We furthermore note that when $\alpha>0$, we have

$$
\frac{d}{d x} U_{\alpha}(x)=U_{\alpha-1}(x) \text {. }
$$

The function $U_{\alpha}(x)$ can clearly for $x \neq 0, \pm 2 \pi, \pm 4 \pi, \ldots$, be uniquely defined also for $\alpha \leq-1$ if we repeatedly utilize the relation (36). We shall in section 6 briefly indicate an important area of applications where the function $U_{\alpha}(x)$ can be successfully applied for some non-integer values of $\alpha$. Due to their importance in applications for partial differential equations in complex geometries both in two and three spatial dimensions [11], however, the piecewise smooth functions (2) obtained for integer values of the parameter $\alpha$, will be at our focus in the rest of this paper.

\section{Removal of Singularities}

Unless a given $2 \pi$-periodic function $w(x)$ is a member of the class $S_{2 \pi}^{\alpha}$ for some relatively large $\alpha$, neither the estimates established in section 2, nor numerical experience give any evidence that truncated or interpolated Fourier series give particularly accurate approximations for $w(x)$. As we shall see in the following, however, such series may still provide valuable tools for handling special subclasses of functions $w \in S_{2 \pi}^{\alpha}$ when $\alpha$ is not large. Normally we will here assume that $\alpha \geq 0$, but in special circumstances we may get useful results also when $\alpha<0$.

The key to the success of Fourier methods for singular functions, is the assumption that the given function $w \in S_{2 \pi}^{\alpha}$ is singular only at a finite number of points $x=\gamma_{j}, j=1,2, \ldots, M$ on the interval $[0,2 \pi]$, and that it can be represented on the form (5), i.e. that for any given integer $Q \geq 0$ we can write

$$
w(x)=w^{Q}(x)+\sum_{n=0}^{Q} \sum_{j=1}^{M} A_{j}^{n} V_{n}\left(x ; \gamma_{j}\right) .
$$

In this paper we shall assume that the location of the singularities $\gamma_{1}, \ldots, \gamma_{M}$ are known with sufficient accuracy. For cases where $\gamma_{1}, \ldots, \gamma_{M}$ are not known, we refer to [10] for algorithms which can be used to determine those locations. For each $j=1, \ldots, M$ and each $n=0,1,2, \ldots$, the special feature function $V_{n}\left(x ; \gamma_{j}\right)$ is assumed to belong to the class $S_{2 \pi}^{\alpha+n}$. From (14), (22) it therefore follows that as long as $\alpha+n>p$ we have

$$
\left\|\frac{d^{p} V_{n}\left(x ; \gamma_{j}\right)}{d x^{p}}-\frac{d^{p} I_{N} V_{n}\left(x ; \gamma_{j}\right)}{d x^{p}}\right\|=O_{s}\left(N^{p-\alpha-n}\right) \quad \text { as } \quad N \rightarrow \infty .
$$

We shall in addition assume that with the possible exception of the points $x=$ $\gamma_{j}+2 m \pi, m=0, \pm 1, \pm 2, \ldots$, the function $V_{n}\left(x ; \gamma_{j}\right)$ is smooth everywhere and such that at any given point $x \neq \gamma_{j}+2 m \pi$ we have for arbitrary $p$

$$
\left|\frac{d^{p} V_{n}\left(x ; \gamma_{j}\right)}{d x^{p}}-\frac{d^{p} I_{N} V_{n}\left(x ; \gamma_{j}\right)}{d x^{p}}\right|=O_{s}\left(N^{p-\alpha-n}\right) \quad \text { as } \quad N \rightarrow \infty .
$$

We note that the above assumptions are satisfied if the special feature functions are given either by $V_{n}\left(x ; \gamma_{j}\right)=U_{\alpha_{j}+n}\left(x-\gamma_{j}\right)$ or by $V_{n}\left(x ; \gamma_{j}\right)=U_{\alpha_{j}+n}\left(\gamma_{j}-x\right)$ for 
some $\alpha_{j} \geq \alpha$, where $U_{\alpha} \in S_{2 \pi}^{\alpha}$ is the special family of functions introduced in the preceding section.

Finally, we shall assume that $V_{n}\left(x ; \gamma_{j}\right)$ possess prototype singularities of the same kind that $w(x)$ is known to have at the point $x=\gamma_{j}$. By this we shall mean that for certain constants $A_{j}^{n}$ (i.e. strengths or amplitudes of the singularities), the function $w^{Q}(x)$ in (37) is a member of the class $S_{2 \pi}^{\alpha+1+Q}$ for each $Q \geq 0$. In order to simplify the construction for cases where the given function $w(x)$ has a relatively complicated structure at the singularities, we may introduce the convention that it is not necessary to exclude the possibility that $\gamma_{j}=\gamma_{l}$ for some $j \neq l$. This may in particular make it easier to handle cases where various special features of different nature simultaneously occur at the same point.

With the above assumptions, it follows from (14), (22) that as long as $\alpha+1+Q>$ $m$ we have

$$
\left\|\frac{d^{m} w^{Q}(x)}{d x^{m}}-\frac{d^{m} I_{N} w^{Q}(x)}{d x^{m}}\right\|=O_{s}\left(N^{m-\alpha-1-Q}\right) \quad \text { as } \quad N \rightarrow \infty .
$$

Furthermore, we observe that (37) is an identity when the proper function $w^{Q}(x)$ and amplitudes $A_{j}^{n}$ have been substituted. If the functions involved are defined (and finite) at the collocation points (16), we therefore get the following relations between the associated discrete Fourier coefficients (17)

$$
\tilde{w}_{k}=\widetilde{\left(w^{Q}\right)_{k}}+\sum_{n=0}^{Q} \sum_{j=1}^{M} A_{j}^{n} \widetilde{\left(V_{n}\right)_{k}}\left(\gamma_{j}\right) .
$$

For the associated interpolated Fourier series (18), we consequently get the identity

$$
I_{N} w(x)=I_{N} w^{Q}(x)+\sum_{n=0}^{Q} \sum_{j=1}^{M} A_{j}^{n} I_{N} V_{n}\left(x ; \gamma_{j}\right) .
$$

By combining (37) and (42) we thus have the identity

$$
w(x)=I_{N} w(x)+\sum_{n=0}^{Q} \sum_{j=1}^{M} A_{j}^{n}\left[V_{n}\left(x ; \gamma_{j}\right)-I_{N} V_{n}\left(x ; \gamma_{j}\right)\right]+w^{Q}(x)-I_{N} w^{Q}(x) .
$$

At least when $x \neq \gamma_{1}, \gamma_{2}, \ldots, \gamma_{M}$, we therefore also have the following identities for $m=0,1,2, \ldots$.

$$
\begin{gathered}
\frac{d^{m}}{d x^{m}} w(x)=\frac{d^{m}}{d x^{m}} I_{N} w(x)+\sum_{n=0}^{Q} \sum_{j=1}^{M} A_{j}^{n}\left[\frac{d^{m}}{d x^{m}} V_{n}\left(x ; \gamma_{j}\right)-\frac{d^{m}}{d x^{m}} I_{N} V_{n}\left(x ; \gamma_{j}\right)\right] \\
+\frac{d^{m}}{d x^{m}} w^{Q}(x)-\frac{d^{m}}{d x^{m}} I_{N} w^{Q}(x) .
\end{gathered}
$$

Thus when $x \neq \gamma_{1}, \gamma_{2}, \ldots, \gamma_{M}$, we conclude from (40), (44) that for $m=0,1,2, \ldots$, we have

$$
\begin{gathered}
\frac{d^{m}}{d x^{m}} w(x)=\frac{d^{m}}{d x^{m}} I_{N} w(x)+\sum_{n=0}^{Q} \sum_{j=1}^{M} A_{j}^{n}\left[\frac{d^{m}}{d x^{m}} V_{n}\left(x ; \gamma_{j}\right)-\frac{d^{m}}{d x^{m}} I_{N} V_{n}\left(x ; \gamma_{j}\right)\right] \\
+O_{s}\left(N^{m-\alpha-1-Q}\right) \quad \text { as } \quad N \rightarrow \infty
\end{gathered}
$$


As a consequence of (20), (37), (41), we furthermore note that the following relations between the discrete and the exact Fourier coefficients hold

$$
\begin{gathered}
\hat{w}_{k}={\widehat{\left(w^{Q}\right)_{k}}}+\sum_{n=0}^{Q} \sum_{j=1}^{M} A_{j}^{n} \widehat{\left(\widehat{V_{n}}\right)_{k}}\left(\gamma_{j}\right) \\
=\tilde{w}_{k}+\sum_{n=0}^{Q} \sum_{j=1}^{M} A_{j}^{n}\left[\widehat{\left(V_{n}\right)_{k}}\left(\gamma_{j}\right)-\widetilde{\left(V_{n}\right)_{k}}\left(\gamma_{j}\right)\right]+{\widehat{\left(w^{Q}\right)_{k}}}^{-\widetilde{\left(w^{Q}\right)_{k}}} \\
(46)=\tilde{w}_{k}+\sum_{n=0}^{Q} \sum_{j=1}^{M} A_{j}^{n}\left[{\widehat{\left(V_{n}\right)_{k}}}_{k}\left(\gamma_{j}\right)-\widetilde{\left(V_{n}\right)_{k}}\left(\gamma_{j}\right)\right]+O_{s}\left(N^{-\alpha-1-Q}\right) \text { as } N \rightarrow \infty .
\end{gathered}
$$

If $Q$ and $N$ have been chosen sufficiently large, the last $O_{s}$-terms in (45) and (46) can be neglected. The relations (45), (46) are therefore suitable for approximation purposes when we are able to calculate all the terms occurring on the right hand side. Since all the functions $V_{n}\left(x ; \gamma_{j}\right)$ by assumption are explicitly known (at least approximately, with high accuracy), and the function $w(x)$ is known at the collocation points (16), the only remaining unknown quantities on the right hand side in (45), (46) are the amplitudes $A_{j}^{n}$.

In general the amplitudes $A_{j}^{n}$ can only be determined approximately, and we shall in the following section describe several procedures for doing this, depending on what additional information that is available for the particular problem at hand. In this connection we note that with the introduced assumption (39), the order of accuracy in (45), (46) will be preserved if approximations $\bar{A}_{j}^{n}$ satisfying

$$
\bar{A}_{j}^{n}=A_{j}^{n}+O_{s}\left(N^{n-Q-1}\right) \quad \text { as } N \rightarrow \infty
$$

are substituted for $A_{j}^{n}$.

We also note that in view of (42), the relation (44) and hence also (45), is equivalent to the relation (6) given in the introduction. Furthermore, we note that if we restrict ourselves to piecewise smooth functions $w(x)$, we may take $V_{n}\left(x ; \gamma_{j}\right)=$ $U_{n}\left(x-\gamma_{j}\right)$. In this case all estimates given in this section are valid when we replace $O_{s}$ with the slightly sharper ordering $O$. This in particular means that with the notation introduced in [11], we have actually proved that the method described in [11] will work in general also when $Q_{1}=Q$ with an accuracy which is of the order that we observed in the numerical computations reported in [11].

\section{Calculation of the singularity amplitudes}

In this section we are going to establish equations for the approximate determination of the amplitudes $A_{j}^{n}$ in the representation (37) for a singular function $w(x)$ given at the collocation points (16). The accuracy of the determined amplitudes will in all cases be at least consistent with (47), but for many problems this will not be sufficient for obtaining a stable and/or robust algorithm. After establishing a system of equations valid for the general case in $\S 5.1$, we shall therefore in $\S 5.2$ establish additional equations valid when detailed knowledge of the function $w(x)$ is available on a certain subinterval of $[0,2 \pi]$. As an example of the latter, we may mention the cases considered in [11] where a buffer zone with $w(x) \equiv 0$ is introduced outside the domain of interest in order to be able to handle problems with complex geometry. 
For special problems it may be possible to establish additional equations for the amplitudes by utilizing the special structure of the problem at hand. This may make it easier to construct a more stable and more robust method, as well as reduce the computational cost involved in applications of the method, thus making the approach more competitive relative to alternative methods. For the special case of piecewise smooth functions we shall in $\S 5.3$ see how finite difference formulas can provide such additional equations.

5.1. Approximate equations for the amplitudes. For an arbitrarily given $\gamma$, the function $V_{n}(x ; \gamma)$ belongs by assumption to the class $S_{2 \pi}^{\alpha+n}$ for $n=0,1, \ldots$. From (11) we therefore have

$$
\widehat{\left(V_{n}\right)}(\gamma)=O_{s}\left(|k|^{-\alpha-n}\right) \quad \text { as }|k| \rightarrow \infty
$$

while from (20) we have

$$
\widetilde{\left(V_{n}\right)_{k}}(\gamma)=\widehat{\left(V_{n}\right)_{k}}(\gamma)+O_{s}\left(N^{-\alpha-n}\right) \quad \text { as } N \rightarrow \infty .
$$

If we let the integer $P>0$ be fixed and let $N-P \leq|k| \leq N$, it follows from (48), (49) that

$$
\widetilde{\left(V_{n}\right)_{k}}(\gamma)=O_{s}\left(N^{-\alpha-n}\right) \quad \text { as } N \rightarrow \infty .
$$

Since by assumption $w^{Q} \in S_{2 \pi}^{\alpha+1+Q}$, it follows in the same way that when $N-P \leq$ $|k| \leq N$, we have for $Q=0,1,2, \ldots$,

$$
\widetilde{\left(w^{Q}\right)_{k}}=O_{s}\left(N^{-\alpha-1-Q}\right) \quad \text { as } N \rightarrow \infty .
$$

From (41), (51) we now get when $N-P \leq|k| \leq N$

$$
\tilde{w}_{k}-\sum_{n=0}^{Q} \sum_{j=1}^{M} A_{j}^{n} \widetilde{\left(V_{n}\right)_{k}}\left(\gamma_{j}\right)=\widetilde{\left(w^{Q}\right)_{k}}=O_{s}\left(N^{-\alpha-1-Q}\right) \quad \text { as } N \rightarrow \infty .
$$

For $Q$ and $N$ sufficiently large, we may therefore neglect the right hand side in (52) and have consequently obtained a system of approximate equations for the amplitudes $A_{j}^{n}$. As partly discussed in [10], [11], the system of equations obtained from (52) may be ill-conditioned and in some cases singular, it may therefore be advantageous to consider the least squares solution of the over-determined system obtained from (52) with $2 P+2>M(Q+1)$

$$
\sum_{|k|=N-P}^{N}\left|\tilde{w}_{k}-\sum_{n=0}^{Q} \sum_{j=1}^{M} A_{j}^{n} \widetilde{\left(V_{n}\right)_{k}}\left(\gamma_{j}\right)\right|^{2} \rightarrow \text { minimum . }
$$

In this way the conditioning of the resulting system of equations for the amplitudes $A_{j}^{n}$ is normally improved, and if we choose $P \geq M(Q+1)-1$, we will in most cases be guaranteed a system which at least in principle is linearly independent and therefore determines the amplitudes uniquely. In section 7 we shall illustrate various aspects related to this issue by considering some numerical examples.

In many cases the solution of (53) will be more accurate than indicated by the above estimates. In fact, the estimate (48) necessarily follows by (11), but (48) is not sufficient to ensure that $V_{n}(x ; \gamma)$ belongs to the class $S_{2 \pi}^{\alpha+n}$. If for instance the 
function $V_{n}(x ; \gamma)$ is given by either $U_{\alpha+n}(x-\gamma)$ or by $U_{\alpha+n}(\gamma-x)$, it follows from (29) that

$$
\widehat{\left(V_{n}\right)_{k}}(\gamma)=O\left(|k|^{-\alpha-n-1}\right) \quad \text { as }|k| \rightarrow \infty .
$$

When $\alpha+n>0$, we have by (19)

$$
{\widetilde{\left(V_{n}\right)_{k}}}_{(\gamma)}={\widehat{\left(V_{n}\right)_{k}}}_{k}(\gamma)+\sum_{m=1}^{+\infty}\left[\widehat{\left(V_{n}\right)_{k+m G}}(\gamma)+{\widehat{\left(V_{n}\right)_{k-m G}}}_{k}(\gamma)\right],
$$

which together with (54) can be used in the same way as in $[11, \S 4]$ to show that

$$
\widetilde{\left(V_{n}\right)_{k}}(\gamma)=\widehat{\left(V_{n}\right)_{k}}(\gamma)+O\left(N^{-\alpha-n-1}\right) \quad \text { as } N \rightarrow \infty .
$$

For fixed $P$, we now get from (54), (56) when $N-P \leq|k| \leq N$ that

$$
\widetilde{\left(V_{n}\right)_{k}}(\gamma)=O\left(N^{-\alpha-n-1}\right) \quad \text { as } N \rightarrow \infty .
$$

As a consequence of (54) and the assumptions made in the previous section, it is clear that for the case considered here we are restricted to functions $w \in S_{2 \pi}^{\alpha}$ satisfying

$$
\hat{w}_{k}=O\left(|k|^{-\alpha-1}\right) \quad \text { as }|k| \rightarrow \infty
$$

and hence

$$
\tilde{w}_{k}=\hat{w}_{k}+O\left(N^{-\alpha-1}\right) \quad \text { as } N \rightarrow \infty .
$$

Since by assumption we have $w^{Q} \in S_{2 \pi}^{\alpha+1+Q}$ for every $Q=0,1, \ldots$, it follows from (41), (51) and (57) that when $N-P \leq|k| \leq N$

$$
\begin{gathered}
\widetilde{\left.\left(w^{Q}\right)_{k}=\widetilde{\left(w^{Q+2}\right.}\right)_{k}}+\sum_{j=1}^{M}\left[A_{j}^{Q+1} \widetilde{\left(V_{Q+1}\right)_{k}}\left(\gamma_{j}\right)+A_{j}^{Q+2}\left(\widetilde{\left.V_{Q+2}\right)_{k}}\left(\gamma_{j}\right)\right]\right. \\
=O\left(N^{-\alpha-Q-2}\right) \quad \text { as } N \rightarrow \infty .
\end{gathered}
$$

In this case therefore, the right hand side in $(52)$ has been shown to be of the order $O\left(N^{-\alpha-Q-2}\right)$ as $N \rightarrow \infty$. This is clearly a sharper estimate than the generally valid estimate given in (52), the solution of (53) can therefore be expected to be quite accurate. This has already been partly confirmed by the numerical results presented in [11], and further numerical examples will be presented in section 7.

5.2. Additional equations when there is a buffer zone. In this subsection we shall establish additional equations valid when we have detailed knowledge of the function $w \in S_{2 \pi}^{\alpha}$ in some subinterval of $[0,2 \pi]$, say the subinterval $(a, b)$. As an example of the latter, a buffer zone with $w(x) \equiv 0$ was in [11] introduced outside the domain of interest in order to be able to handle problems with complex geometry. There is clearly no loss of generality by assuming that $w(x) \equiv 0$ on the subinterval $(a, b)$ since it is always possible to subtract any other given value the function may have there. With that assumption, we therefore get from (43) that everywhere in $(a, b)$ we have

$$
\begin{gathered}
I_{N} w(x)+\sum_{n=0}^{Q} \sum_{j=1}^{M} A_{j}^{n}\left[V_{n}\left(x ; \gamma_{j}\right)-I_{N} V_{n}\left(x ; \gamma_{j}\right)\right] \\
=O_{s}\left(N^{-\alpha-1-Q}\right) \quad \text { as } \quad N \rightarrow \infty .
\end{gathered}
$$


At those of the collocation points (16) which are located in the subinterval $(a, b)$, the left hand side in (61) will for arbitrary amplitudes $A_{j}^{n}$ either nearly vanish or actually vanish, depending on whether $G$ is even or odd, respectively. In between the collocation points in $(a, b)$, however, the left hand side in (61) will normally only be small when quite accurate values for the amplitudes $A_{j}^{n}$ have been substituted. As a consequence, additional approximate equations for the amplitudes $A_{j}^{n}$ can be obtained by equating the left hand side in (61) to zero at one or more noncollocation points in $(a, b)$. These equations can then either be used in conjunction with (52) to make a slightly larger least squares problem than (53), or alternatively, as a stand alone system if sufficiently many independent non-collocation points have been chosen in $(a, b)$.

In addition to (61), the relation (45) with the assumption that $w(x) \equiv 0$ on $(a, b)$, shows that on $(a, b)$ we also have for $m=1,2, \ldots$

$$
\begin{gathered}
\frac{d^{m}}{d x^{m}} I_{N} w(x)+ \\
+\sum_{n=0}^{Q} \sum_{j=1}^{M} A_{j}^{n}\left[\frac{d^{m}}{d x^{m}} V_{n}\left(x ; \gamma_{j}\right)-\frac{d^{m}}{d x^{m}} I_{N} V_{n}\left(x ; \gamma_{j}\right)\right] \\
=O_{s}\left(N^{m-\alpha-1-Q}\right) \quad \text { as } \quad N \rightarrow \infty .
\end{gathered}
$$

In contrast to (61), the relations (62) will not automatically be satisfied at the collocation points. We are therefore here more free than for (61) to choose where we want to put the left hand side in (62) equal to zero. Normally, the relations (62) will at an arbitrarily chosen point in $(a, b)$ provide us with additional approximate equations for the amplitudes $A_{j}^{n}$. We may for instance choose the boundary points $x=a^{+}$and $x=b^{-}$, or any other convenient point inside the interval $(a, b)$. With this flexibility, it is not difficult to obtain an independent stand alone system which in principle determines all the amplitudes $A_{j}^{n}$ uniquely. In order to get a balanced system of equations, the error terms on the right hand side in (61), (62) clearly indicate that (62) should be multiplied by a weight-factor proportional to $N^{-m}$ before approximate solutions are sought.

Since the obtained system normally can be expected to be ill-conditioned, the least squares problem for an enlarged system which for instance also includes some of the generally valid equations deduced in $\S 5.1$, may lead to a better conditioned system and may thus be more feasible in practice. The most favorable choice of system will clearly depend on the actual problem at hand, but the inherent flexibility should make it possible in many cases to establish systems which determine the amplitudes with sufficient accuracy. An application of this construction has already been seen to be feasible for the solution of the heat equation in complex geometries [14], and further illustrating examples will be given in section 7 .

5.3. Utilization of finite differences. For the important special case of piecewise smooth functions where $V_{n}\left(x, \gamma_{j}\right)$ is given by (2), the amplitudes $A_{j}^{n}$ are given by (3). Since the singularity locations $\gamma_{j}$ need not be grid points, we will in general have to deal with nonuniform grids if finite difference formulas shall be employed in the determination of the right hand side of (3). In principle, however, such finite difference formulas of arbitrary order are known and can be set up for derivatives of any given order. Thus finite difference formulas can in principle be set up for all the unknown amplitudes $A_{j}^{n}$ in this case, formally satisfying (47). Such high order finite difference formulas are known to lack robustness, however, they will therefore 
normally be unsuitable for our purpose here. In the words of Lyness [21, p.101], such finite difference approximations are notoriously unreliable.

Even with this background there is still one combination of such finite difference formulas which may be suitable for our purpose for cases where there is one or more buffer zones. The combination in question here is perhaps better known as a Taylor expansion near each singularity location which is next to a buffer zone. In fact, if there is a buffer zone with $w(x) \equiv 0$ to the left of the singularity location $\gamma_{j}$, we have $w^{(n)}\left(\gamma_{j}^{-}\right)=0$ for every $n=0,1,2, \ldots$, and (3) consequently implies that

$$
A_{j}^{n}=\frac{d^{n} w}{d x^{n}}\left(\gamma_{j}^{+}\right)
$$

For $x>\gamma_{j}$, a Taylor expansion therefore results in

$$
w(x)=A_{j}^{0}+\left(x-\gamma_{j}\right) A_{j}^{1}+\cdots+\frac{\left(x-\gamma_{j}\right)^{Q}}{Q !} A_{j}^{Q}+O\left(\left|x-\gamma_{j}\right|^{Q+1}\right) .
$$

If we let $x_{k}$ denote the grid-point next to $\gamma_{j}$ on the right, i.e. if $x_{k-1} \leq \gamma_{j}<x_{k}$, then (64) with $x=x_{k}$ clearly gives us an approximate equation for the amplitudes $A_{j}^{0}, \ldots, A_{j}^{Q}$ with an error term which is of the order $O\left(N^{-Q-1}\right)$ as $N \rightarrow \infty$. Although the error term normally grows if we take (64) with $x=x_{k+1}$, the error term is still of the order $O\left(N^{-Q-1}\right)$, thus providing us with an additional equation. If the distance to the next singularity location is large enough, this process can formally be continued to also include the grid-points $x_{k+2}, x_{k+3}, \ldots, x_{k+Q}$, thus providing us with a closed system for the determination of the amplitudes $A_{j}^{0}, \ldots, A_{j}^{Q}$. The solution of this system is actually a set of finite difference formulas which formally are of the appropriate order (47). Our numerical experiments do show, however, that if more than 2 or possibly 3 such equations are considered, a loss in robustness and accuracy can be expected, indicating that only a few of these equations should be considered as part of a larger system incorporating equations obtained earlier in this section.

Except for a change in sign, a similar construction applies at a singularity location $\gamma_{l}$ which has a buffer zone to the right. In fact, since we then have $w^{(n)}\left(\gamma_{l}^{+}\right)=0$ for every $n=0,1,2, \ldots$, equation (3) now implies that

$$
A_{l}^{n}=-\frac{d^{n} w}{d x^{n}}\left(\gamma_{l}^{-}\right) \text {. }
$$

For $x<\gamma_{l}$, a Taylor expansion consequently results in

$$
w(x)=-A_{l}^{0}-\left(x-\gamma_{l}\right) A_{l}^{1}-\cdots-\frac{\left(x-\gamma_{l}\right)^{Q}}{Q !} A_{l}^{Q}+O\left(\left|x-\gamma_{l}\right|^{Q+1}\right) .
$$

If we let $x_{m}$ denote the grid-point next to $\gamma_{l}$ on the left, i.e. if $x_{m}<\gamma_{l} \leq x_{m+1}$, then (66) with $x=x_{m}, x_{m-1}$ and possibly also $x=x_{m-2}$, clearly give us two (or possibly three) approximate equations for the amplitudes $A_{l}^{0}, \ldots, A_{l}^{Q}$ with an error term which is of the order $O\left(N^{-Q-1}\right)$ as $N \rightarrow \infty$.

\section{Some APplications}

The area of application which we primarily have had in mind in this paper, is the solution of partial differential equations in complex geometries utilizing an ordinary, fixed, uniform, Cartesian system of grid-points [11]. The derivative with respect to $x$ at each of those grid-points then clearly depends only on the behavior of the function on the grid-line through that point where $y=$ constant (and $z=$ constant 
etc., if the dimension is higher than 2). Similarly, the derivative with respect to $y$ depends only on the grid-line where $x=$ constant, $z=$ constant, etc. etc. The corresponding one-dimensional function on each grid-line is relevant for our problem only on certain disjoint subintervals $\left[\gamma_{1}, \gamma_{2}\right],\left[\gamma_{3}, \gamma_{4}\right], \ldots,\left[\gamma_{M-1}, \gamma_{M}\right]$, which for some $M \geq 2$ are uniquely determined by the geometry of the problem. Points outside the subintervals $\left[\gamma_{1}, \gamma_{2}\right],\left[\gamma_{3}, \gamma_{4}\right], \ldots,\left[\gamma_{M-1}, \gamma_{M}\right]$ correspond to points outside the domain of interest, and are therefore irrelevant for our problem. The subintervals $\left[\gamma_{1}, \gamma_{2}\right],\left[\gamma_{3}, \gamma_{4}\right], \ldots,\left[\gamma_{M-1}, \gamma_{M}\right]$ may clearly vary from grid-line to grid-line, and may also vary in time if the geometry of our problem is time dependent.

Since the derivative of the function on each of the subintervals is independent of the behavior of the function on the other subintervals, each subinterval can be handled separately. Thus, in order to handle the space discretization problem on a Cartesian grid for problems in complex geometries, it clearly suffices to design a SUBROUTINE that accurately calculates the derivative of an arbitrarily given one-dimensional smooth function on a uniform set of grid-points in an arbitrarily given interval $\left[\gamma_{1}, \gamma_{2}\right]$ utilizing only the values of the function at the same grid points and the relevant boundary conditions at the endpoints $\gamma_{1}, \gamma_{2}$ of the interval. These endpoints $\gamma_{1}, \gamma_{2}$ will normally not coincide with grid-points. There is clearly no essential loss of generality by assuming that $\left[\gamma_{1}, \gamma_{2}\right]$ is a subinterval of $[0,2 \pi]$, and that the rest of the interval $[0,2 \pi]$ is a buffer zone. Thus all the machinery developed earlier in this paper for calculating the derivative of a piecewise smooth function can be applied in the construction of such a SUBROUTINE. Some numerical results obtained for such constructions have already been published in [11], [14], and improved results based on the additional insight gained in this paper will be given in the following section. Applications to partial differential equations will be given elsewhere. We would here like to add that, in principle, the same type of approach can also be applied to problems involving shocks in the solution, if the location of the shocks can be accurately calculated. In fact, the location of the shock can then be handled as an additional boundary (possibly with appropriate shock relations as boundary conditions) manifesting itself in appropriate values of $\gamma_{j}$ on the various grid-lines. For one-dimensional problems, some such results obtained by an analogous modified Galerkin method have been published in [9], [12], [13].

Another area where interesting applications can be given is numerical quadrature. If the integral over the interval $[0,2 \pi]$ of a one-dimensional function $w(x)$ is needed, it is from (8) given by $2 \pi \hat{w}_{0}$, which clearly can be accurately approximated by the expressions given in (46). If in particular $w(x)$ is smooth on $[0,2 \pi]$, the $2 \pi$-periodic extension of $w(x)$ is in general only piecewise smooth with one jumpsingularity in each period (i.e. we may take $M=1$ and $\gamma_{1}=0$ ). In this case it is not difficult to show that (46) results in a quadrature formula which is equivalent to the classical Euler-Maclaurin formula [7], [21].

The formula (46) can clearly also be utilized to obtain generalized EulerMaclaurin quadrature formulas in cases where the function $w(x)$ has various types of additional singularities on the interval $[0,2 \pi]$. We shall here only look more closely at the formula we obtain when the integral over a subinterval $\left[\gamma_{1}, \gamma_{2}\right] \subset[0,2 \pi]$ of a smooth function $w(x)$ is needed. We may then assume that $w(x)$ vanishes identically on the rest of the interval $[0,2 \pi]$, providing us with a buffer zone. With these assumptions, $w(x)$ is piecewise smooth, and $V_{n}(x, \gamma)=U_{n}(x-\gamma)$ is given by (2). By arguments analogous to those given in the last part of section 5.1, it is easy to show that the error term in (46) then actually is of the order $O\left(N^{-Q-2}\right)$ as $N \rightarrow \infty$ 
instead of $O_{s}\left(N^{-\alpha-1-Q}\right)$. In view of (4) we therefore get the following formula for this case

$$
\begin{aligned}
\int_{\gamma_{1}}^{\gamma_{2}} w(x) d x & =2 \pi \hat{w}_{0} \\
& =2 \pi \tilde{w}_{0}-2 \pi \sum_{n=0}^{Q} \sum_{j=1}^{2} A_{j}^{n} \widetilde{\left(V_{n}\right)_{0}}\left(\gamma_{j}\right)+O\left(N^{-Q-2}\right) \quad \text { as } N \rightarrow \infty .
\end{aligned}
$$

Application of (4) and (19) readily gives for $n \geq 1$

$$
\begin{gathered}
\widetilde{\left(V_{n}\right)_{0}}\left(\gamma_{j}\right)=\sum_{m=1}^{+\infty}\left[\widehat{\left(V_{n}\right)_{m G}}\left(\gamma_{j}\right)+\widehat{\left(V_{n}\right)}-m G\left(\gamma_{j}\right)\right] \\
=\frac{(-1)^{n+1}}{G^{n+1}} \sum_{m=1}^{+\infty}\left[\frac{e^{i m G \gamma_{j}}}{2 \pi(i m)^{n+1}}+\frac{e^{-i m G \gamma_{j}}}{2 \pi(-i m)^{n+1}}\right]=\frac{(-1)^{n+1} U_{n}\left(G \gamma_{j}\right)}{G^{n+1}} .
\end{gathered}
$$

It is not difficult to show that (68) also holds for $n=0$ if we apply the convention that $U_{0}(2 k \pi)=0, k=0, \pm 1, \pm 2, \ldots$, which implies that $U_{0}(x)$ is everywhere given by the sum of its Fourier series. With this convention we have therefore established the following generalization of the Euler-Maclaurin formula

$\int_{\gamma_{1}}^{\gamma_{2}} w(x) d x=2 \pi \tilde{w}_{0}-2 \pi \sum_{n=0}^{Q} \sum_{j=1}^{2} \frac{(-1)^{n+1} U_{n}\left(G \gamma_{j}\right) A_{j}^{n}}{G^{n+1}}+O\left(G^{-Q-2}\right) \quad$ as $\quad G \rightarrow \infty$

where by (3), (16) and (17) we have

$$
A_{1}^{n}=\frac{d^{n} w}{d x^{n}}\left(\gamma_{1}^{+}\right) \quad, \quad A_{2}^{n}=-\frac{d^{n} w}{d x^{n}}\left(\gamma_{2}^{-}\right) \quad, \quad \tilde{w}_{0}=\frac{1}{G} \sum_{l=0}^{G-1} w(2 \pi l / G) .
$$

For the above formulas to be valid also when $\gamma_{1}$ and/or $\gamma_{2}$ are grid-points, it is in view of the convention introduced above for $U_{0}(x)$ necessary to assume that $w(x)$ is also everywhere given by the sum of its Fourier series. Since $w(x)$ by assumption is smooth on the interval $\left[\gamma_{1}, \gamma_{2}\right]$ and vanishes outside $\left[\gamma_{1}, \gamma_{2}\right]$, it suffices here to introduce the convention that $w\left(\gamma_{1}\right)=w\left(\gamma_{1}^{+}\right) / 2$ and $w\left(\gamma_{2}\right)=w\left(\gamma_{2}^{-}\right) / 2$, which is easily seen to be consistent with the assumption that (37) is an identity.

In order to apply the above quadrature formula, the efficient and robust calculation of the amplitudes $A_{1}^{n}, A_{2}^{n}, n=0,1, \ldots, Q$, by the equations established in the preceding section will clearly play a key role. One area where the formulas (69), (70) may seem especially useful is for integration in two or more dimensions, where efficient quadrature formulas are not very well developed [7]. In fact, we shall now see that repeated use of (69), (70) on a Cartesian grid will give us accurate approximations for the double integral of an arbitrarily given smooth function $f(x, y)$ on a domain which is of one of the following two types

$$
\begin{aligned}
& S_{1}=\left\{x, y \mid \gamma_{1} \leq x \leq \gamma_{2} \quad \& \quad \phi_{1}(x) \leq y \leq \phi_{2}(x)\right\} \\
& S_{2}=\left\{x, y \mid \mu_{1} \leq y \leq \mu_{2} \& \quad \psi_{1}(y) \leq x \leq \psi_{2}(y)\right\},
\end{aligned}
$$

where it is assumed that $\phi_{1}(x) \leq \phi_{2}(x)$ are smooth on the interval $\left[\gamma_{1}, \gamma_{2}\right]$, and that $\psi_{1}(y) \leq \psi_{2}(y)$ are smooth on the interval $\left[\mu_{1}, \mu_{2}\right]$. 
In the notation of Apostol [1], $S_{1}$ is a domain of Type I, and $S_{2}$ is a domain of Type II, and the double integral of a smooth function $f(x, y)$ can be evaluated by repeated one-dimensional integration in the following way

$$
I_{1}=\iint_{S_{1}} f(x, y) d x d y=\int_{\gamma_{1}}^{\gamma_{2}} \Phi(x) d x \quad, \quad I_{2}=\iint_{S_{2}} f(x, y) d x d y=\int_{\mu_{1}}^{\mu_{2}} \Psi(y) d y,
$$

where

$$
\Phi(x)=\int_{\phi_{1}(x)}^{\phi_{2}(x)} f(x, y) d y, \Psi(y)=\int_{\psi_{1}(y)}^{\psi_{2}(y)} f(x, y) d x
$$

From the assumptions introduced, it follows that $\Phi(x)$ is smooth on $\left[\gamma_{1}, \gamma_{2}\right]$ and $\Psi(y)$ is smooth on $\left[\mu_{1}, \mu_{2}\right]$. Without essential loss of generality we may assume that both $S_{1}$ and $S_{2}$ are subsets of the square $[0,2 \pi] \times[0,2 \pi]$, which in particular imply that $\left[\gamma_{1}, \gamma_{2}\right] \subset[0,2 \pi]$ and $\left[\mu_{1}, \mu_{2}\right] \subset[0,2 \pi]$. Thus the last one-dimensional form of the integrals $I_{1}, I_{2}$ given by (73) can clearly be approximately evaluated by the formulas $(69),(70)$. Since also $\Phi(x)$ can be approximately evaluated by the formulas (69), (70) for each $x \in\left[\gamma_{1}, \gamma_{2}\right]$, and $\Psi(y)$ can be approximately evaluated by the formulas $(69),(70)$ for each $y \in\left[\mu_{1}, \mu_{2}\right]$, the above assertion that $I_{1}, I_{2}$ can be approximately evaluated by repeated applications of the formulas (69), (70) on a Cartesian grid therefore follows.

It is not difficult to find a domain which does not satisfy all the requirements we have put on $S_{1}$ and $S_{2}$ above. Consider for instance a circular domain $S=$ $\left\{x, y \mid(x-3)^{2}+(y-3)^{2} \leq 4\right\}$, which we also can express in the following alternative forms resembling $S_{1}$ and $S_{2}$

$$
\begin{aligned}
S & =\left\{x, y \mid 1 \leq x \leq 5 \quad \& \quad 3-\sqrt{4-(x-3)^{2}} \leq y \leq 3+\sqrt{4-(x-3)^{2}}\right\}, \\
& =\left\{x, y \mid 1 \leq y \leq 5 \& 3-\sqrt{4-(y-3)^{2}} \leq x \leq 3+\sqrt{4-(y-3)^{2}}\right\} .
\end{aligned}
$$

The intervals associated with $S$ which resemble $\left[\gamma_{1}, \gamma_{2}\right]$ and $\left[\mu_{1}, \mu_{2}\right]$ in (71), (72), are both seen to be $[1,5]$, and the double integral can also in this case be evaluated by repeated one-dimensional integrations as in (73), (74). We do see, however, that smoothness of $f(x, y)$ in this case is not sufficient to guarantee that the corresponding functions $\Phi(x)$ and $\Psi(y)$ are smooth on the interval $[1,5]$. In fact, if we take $f(x, y) \equiv 1$ in $S$ for instance, we readily obtain from (74)

$$
\Phi(x)=2 \sqrt{4-(x-3)^{2}} \quad, \quad \Phi(y)=2 \sqrt{4-(y-3)^{2}} .
$$

At both endpoints of the interval $[1,5], \Phi(x)$ and $\Psi(y)$ are both seen to have singularities of the type (35) with $\alpha=1 / 2$, thus indicating the need for special feature functions of the type $V_{0}(x, 1)=U_{1 / 2}(x-1)$ and $V_{0}(x, 5)=U_{1 / 2}(5-x)$ instead of the usual Bernoulli polynomials (2). It is not difficult to see that for more general piecewise smooth functions with discontinuities along more general curves in the plane, the second performed Fourier transformation (or, equivalently, the first inverse Fourier transformation) will involve singularities which typically can be handled by special feature functions of the following types $V_{n}(x, \gamma)=U_{1 / 2+n}(x-\gamma)$ and $V_{n}(x, \gamma)=U_{1 / 2+n}(\gamma-x), n=0,1, \ldots$. We hope to be able to develop these 
ideas further elsewhere, and applications for instance to the accurate reconstructions of two-dimensional pictures from their two-dimensional Fourier transform may here seem promising in view of the existing one-dimensional version [10]. In addition, we can foresee possibilities for applications both to quadrature and to partial differential equations.

We would finally like to note here that by dividing a two-dimensional domain into a finite number of subdomains, it is normally possible to get each of the subdomains to be either of the type $S_{1}$ given by (71), or of the type $S_{2}$ given by (72), with all assumptions fulfilled. As an example, we can divide the circular domain $S$ given by (75) into three subdomains by the two lines $y=2$ and $y=4$, respectively. Similar constructions are also feasible in higher dimensions. We may therefore conclude that by simple decomposition of the domain, it is normally possible to obtain accurate approximations for multiple integrals over quite general domains by repeated utilization of the quadrature formulas (69), (70) on a Cartesian grid (which does not have to be altered from one sub-domain to the next).

\section{NumericAL EXAMPLES}

In order to illustrate the accuracy which potentially can be achieved by the method we have described in this paper, we shall first look at the accuracy obtained for the first derivative of the following function

$$
v(x)= \begin{cases}0 & \text { when } x \in[0,0.1), \\ \exp (-x) & \text { when } x \in[0.1,4.6], \\ 0 & \text { when } x \in(4.6,2 \pi],\end{cases}
$$

when the exact amplitudes at the two discontinuity locations $\gamma_{1}=0.1$ and $\gamma_{2}=4.6$ are substituted in the subroutine where the derivative at the grid-points (16) is calculated utilizing normal double precision (53 bits). In Figure 1 the RMS error

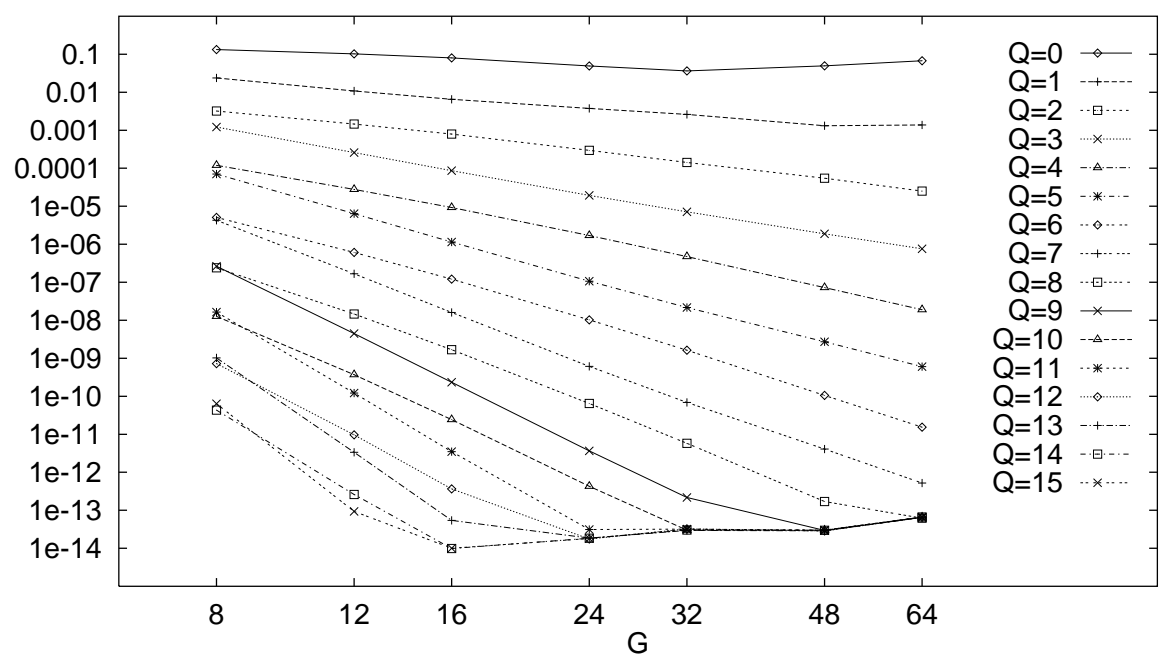

FiguRE 1. RMS error for the derivative of the function (77) with exact amplitudes. 


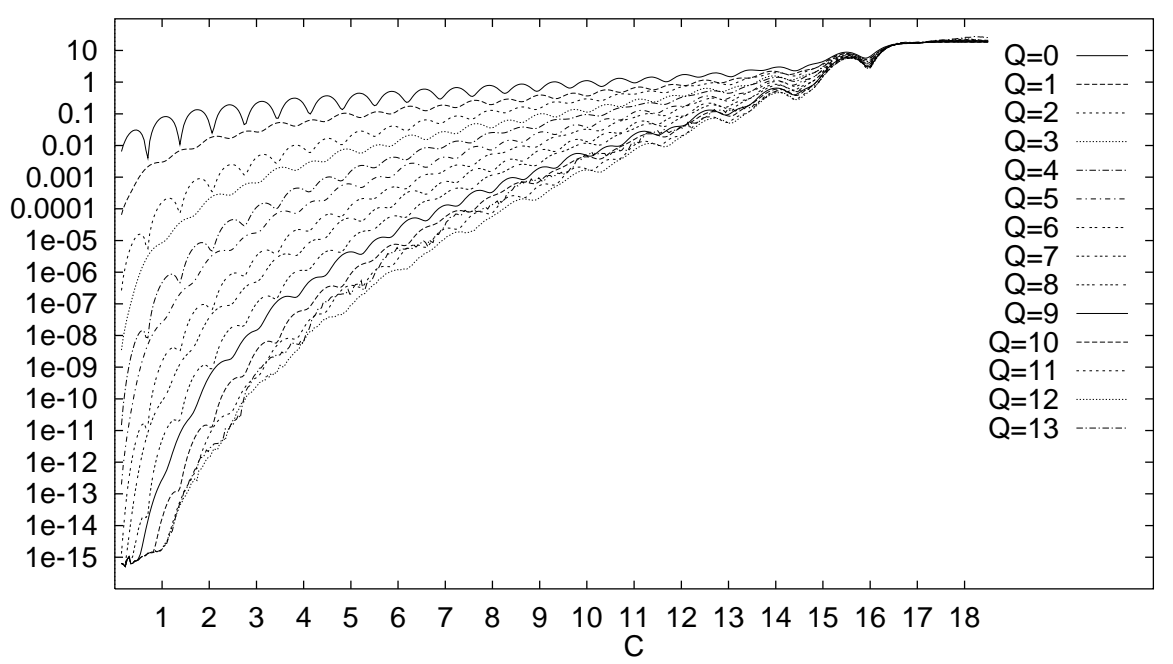

FiguRE 2. RMS error for the derivative of the function (78) with $G=32$ and exact amplitudes, when $\gamma_{1}=0.1, \gamma_{2}=4.55$.

in the derivative at the grid-points is shown for various values of the parameter $Q$, and for different numbers of grid-points $G$ on the interval $[0,2 \pi]$. A plot for the corresponding maximal errors would show a similar behavior. For the largest values of the parameter $Q$, the maximal errors will in fact differ only slightly from those shown in Figure 1. Normally, the maximal errors will be found at the grid-points which are located nearest the discontinuity locations. Away from those locations, the accuracy is usually somewhat better and can actually be further improved by various forms of filtering [23], but we shall not pursue that issue here. The results found are easily seen to be consistent with the estimates given in the preceding sections even when the number of grid-points is small. For other functions with a similar structure we get qualitatively the same type of results. Quantitatively we may get differences due to the different resolution requirements the actual functions may have.

In order to exploit the resolution issue further, we have looked at the accuracy obtained for the first derivative of the following family of functions

$$
w_{c}(x)= \begin{cases}0 & \text { when } x \in\left[0, \gamma_{1}\right), \\ \cos (c x) & \text { when } x \in\left[\gamma_{1}, \gamma_{2}\right] \\ 0 & \text { when } x \in\left(\gamma_{2}, 2 \pi\right]\end{cases}
$$

for various values of the constant $c$, and for various discontinuity locations $\gamma_{1}<\gamma_{2}$ in the interval $[0,2 \pi]$. When the exact amplitudes at the two discontinuity locations are substituted in the subroutine where the derivative at the grid-points (16) is calculated, the behavior of the RMS error is plotted in Figure 2 for the particular choice $\gamma_{1}=0.1, \gamma_{2}=4.55$ when the number of grid-points is 32 . If $\gamma_{1}, \gamma_{2}$ are chosen differently, the overall error will be seen to remain virtually unchanged, only the oscillatory behavior with respect to the parameter $c$ which can be seen on Figure 2, will appear in a different way. In fact, for smaller subintervals $\left[\gamma_{1}, \gamma_{2}\right]$, the 


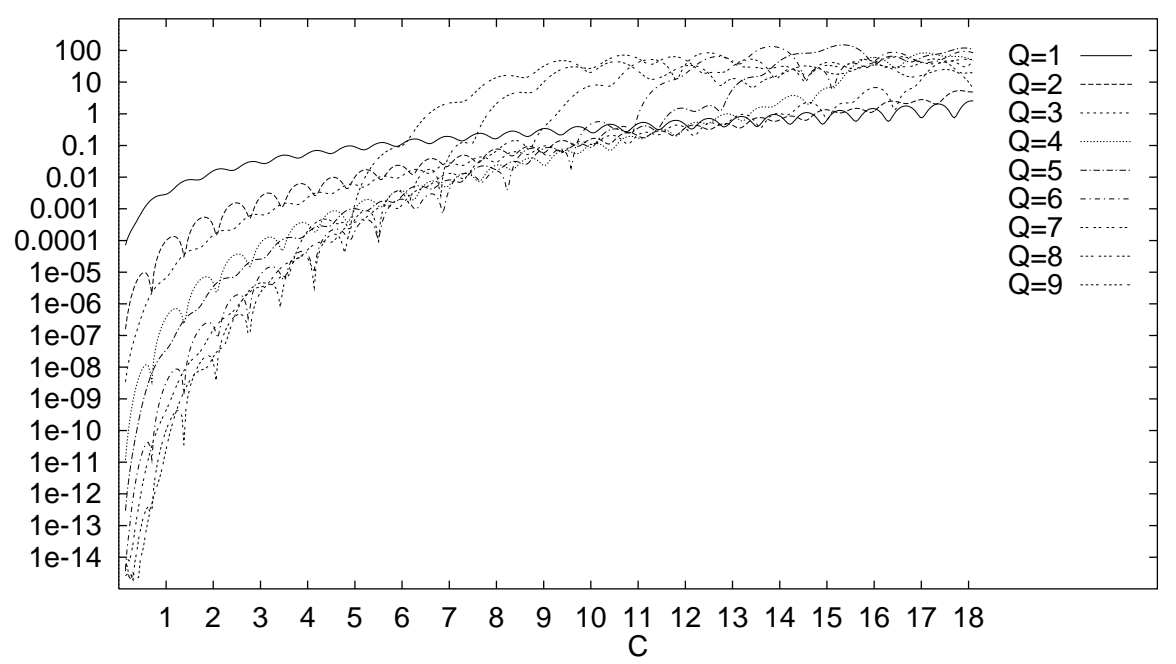

FiguRE 3. RMS error for the derivative of the function (78) with $G=48$ and amplitudes determined by (53) with $P=2 Q-1$ when $\gamma_{1}=0.1, \gamma_{2}=4.55$.

oscillations will disappear, while the oscillations will get more and more pronounced the larger the interval $\left[\gamma_{1}, \gamma_{2}\right] \subset[0,2 \pi]$ is. With this behavior in mind, Figure 2 can therefore be regarded as representative for the accuracy which in principle is obtainable for arbitrarily given values of $\gamma_{1}, \gamma_{2}$. On Figure 2 it seems that $Q=12$ gives the optimal results in most cases for our normal double precision calculations (53 bits).

For the function (78) we see that with 32 grid-points on $[0,2 \pi], c=16$ is corresponding to 2 grid-points pr. wavelength and cannot therefore be expected to give resolution. Already with 2.5 grid-points pr. wavelength, however, which on Figure 2 corresponds to $c=12.8$, the accuracy for the higher values of $Q$ shown is better than $1 \%$. Completely analogous results can be obtained for other values of the locations $\gamma_{1}, \gamma_{2}$, and other numbers of grid-points on the interval $[0,2 \pi]$.

We shall now calculate the derivative of the function (78) at the grid-points (16) in the case where the exact amplitudes are not known in advance. We shall in our first calculations assume, however, that the principal amplitudes $A_{1}^{0}, A_{2}^{0}$ are known, or equivalently, that the limit-values of the function are known at the two boundary points $x=\gamma_{1}$ and $x=\gamma_{2}$, respectively. In the various applications we later shall encounter, the principal amplitudes $A_{1}^{0}, A_{2}^{0}$ will normally either be known from Dirichlet boundary data, or may be calculated separately by for instance letting the two boundary points $x=\gamma_{1}$ and $x=\gamma_{2}$ be treated as two extra grid-points. If the higher order amplitudes $A_{1}^{n}, A_{2}^{n}, n=1, \ldots, Q$, are calculated from (53) with $P=2 Q-1$ utilizing the SVD subroutines from LAPACK [2], we obtain the results plotted in Figure 3.

If the same calculation is done with $P=Q$, we obtain the results plotted in Figure 4.

The first lesson to be learned from Figures 3 and 4 is that for a well resolved function (i.e. for $c$ small), we may obtain better accuracies by utilizing a larger 


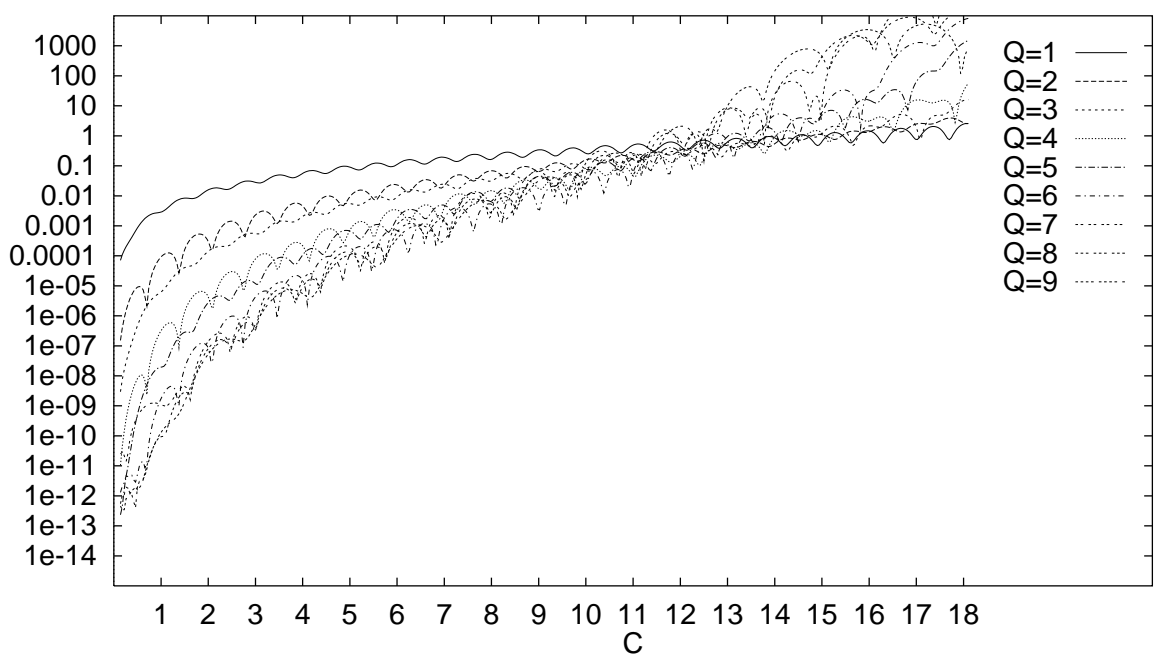

FiguRE 4. RMS error for the derivative of the function (78) with $G=48$ and amplitudes determined by (53) with $P=Q$ when $\gamma_{1}=0.1, \gamma_{2}=4.55$.

part of the spectrum in the determination of the amplitudes. Second, the resolution properties of the algorithm is reduced when a larger part of the spectrum than strictly necessary is utilized in the determination of the amplitudes. Third, large errors stemming from unresolved parts of the function can be expected, as is particularly pronounced in Figure 4. Such errors may lead to lack of robustness, and may perhaps also explain instabilities we have encountered when the algorithm has been applied to solving initial-boundary value problems for partial differential equations. The calculations shown in Figures 3 and 4 are done for 48 grid-points, but completely analogous results can be obtained for other numbers of grid-points on the interval $[0,2 \pi]$, as well as for other choices of the locations $\gamma_{1}, \gamma_{2}$.

For the function (78) there clearly is a buffer zone which can be utilized to set up additional equations for the amplitudes as discussed in sections 5.2 and 5.3. These can then be combined with (53) into a larger over-determined system of equations which then can be solved utilizing the SVD subroutines from LAPACK. An infinite number of different combinations are here clearly possible. When a sufficient number of independent equations (61), (62) are included, we have only observed slight changes in the solutions by including more of the equations (61), (62). The same is not true for the equations set up in sections 5.1 and 5.3. In fact, especially when high resolution is desirable, we have found that it normally is advantageous to limit the number $P$ in (53) to 2 and also limit the Taylor equations from section 5.3 to the 2 internal grid-points (i.e. located in the interval $\left[\gamma_{1}, \gamma_{2}\right]$ ) which are nearest each of the 2 boundary points $\gamma_{1}, \gamma_{2}$.

In the calculations we shall report here, we have evaluated (61), (62) at each boundary point, at the midpoint between the boundary point and the first internal grid-point, at the first internal grid-point, and at the midpoint between the first and the second internal grid-point next to each boundary point. In (62) we have not used $m$ larger than 2 . With this setup we have obtained the accuracies plotted in 


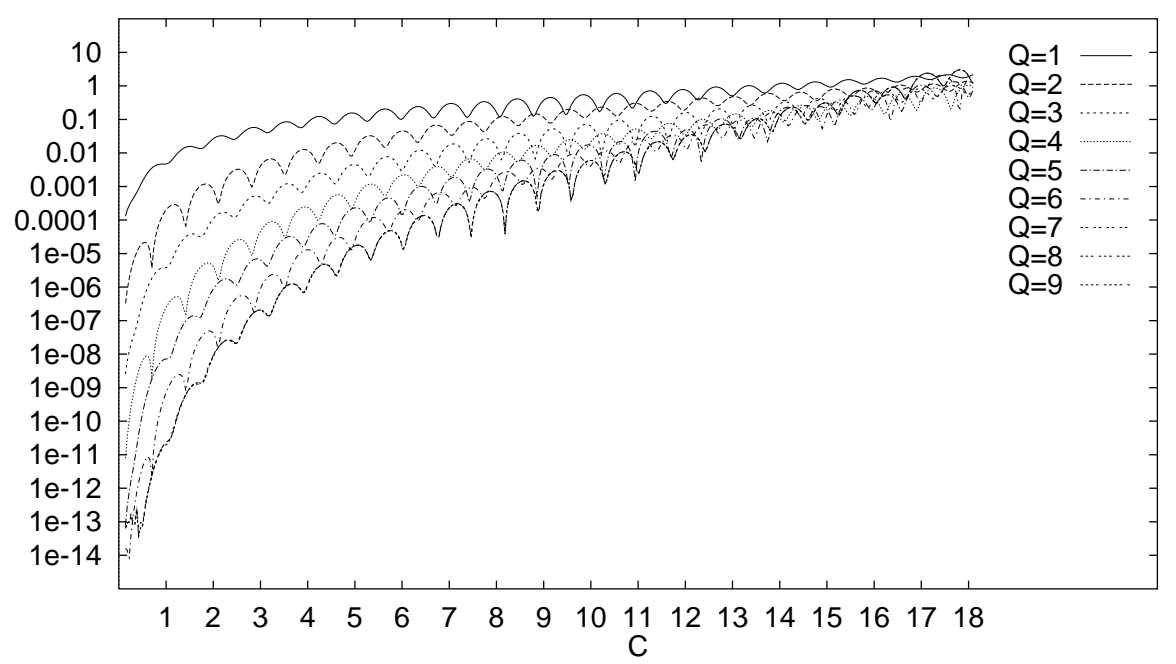

FiguRE 5. RMS error for the derivative of the function (78) with $G=48$ and amplitudes determined by a mixture of the available equations when $\gamma_{1}=0.1, \gamma_{2}=4.55$.

Figure 5 for 48 grid-points in the interval $[0,2 \pi]$ for the specific values of boundary points $\gamma_{1}=0.1, \gamma_{2}=4.55$. Again we find that the overall accuracies depend very little on the locations $\gamma_{1}, \gamma_{2}$ as long as there are at least a handful of internal gridpoints. The observed difference is also here a change in the oscillatory behavior with respect to the parameter $c$, similar to that reported earlier. The general trend that can be deduced from Figure 5, and which also holds for other numbers of gridpoints in the interval $[0,2 \pi]$, is that already with 3 grid-points pr. wavelength the error does not exceed $10 \%$, and with 3.5 grid-points pr. wavelength the error is less than $1 \%$ for the largest feasible values of $Q$. For more grid-points pr. wavelength the error rapidly decreases. We regard these results as very promising indeed. In our calculations we have found that $Q=8$ is optimal for $G=32, Q=7$ is optimal for $G=48$, and $Q=6$ is optimal for $G=64$, but these numbers may come out slightly different for alternative implementations of the equations utilized. Although large errors occur for unresolved functions, the errors are considerably lower than those observed in Figure 4, thus the robustness of the algorithm is also considerably improved.

In Figure 6 we have plotted the results for 48 grid-points in the interval $[0,2 \pi]$ when the "best" set of equations determined above is extended to also determine the principal amplitudes $A_{1}^{0}, A_{2}^{0}$ in addition to the other amplitudes $A_{1}^{n}, A_{2}^{n}, n=$ $1, \ldots, Q$. Analogous calculations have also been carried out with other numbers of grid-points and with other locations $\gamma_{1}, \gamma_{2}$ in the interval $[0,2 \pi]$, with results that are consistent with the general trends discussed earlier. Although the overall accuracy is somewhat reduced compared to results obtained with exact values for the principal amplitudes $A_{1}^{0}, A_{2}^{0}$, we feel that the results are still quite good and should therefore be useful in applications. 


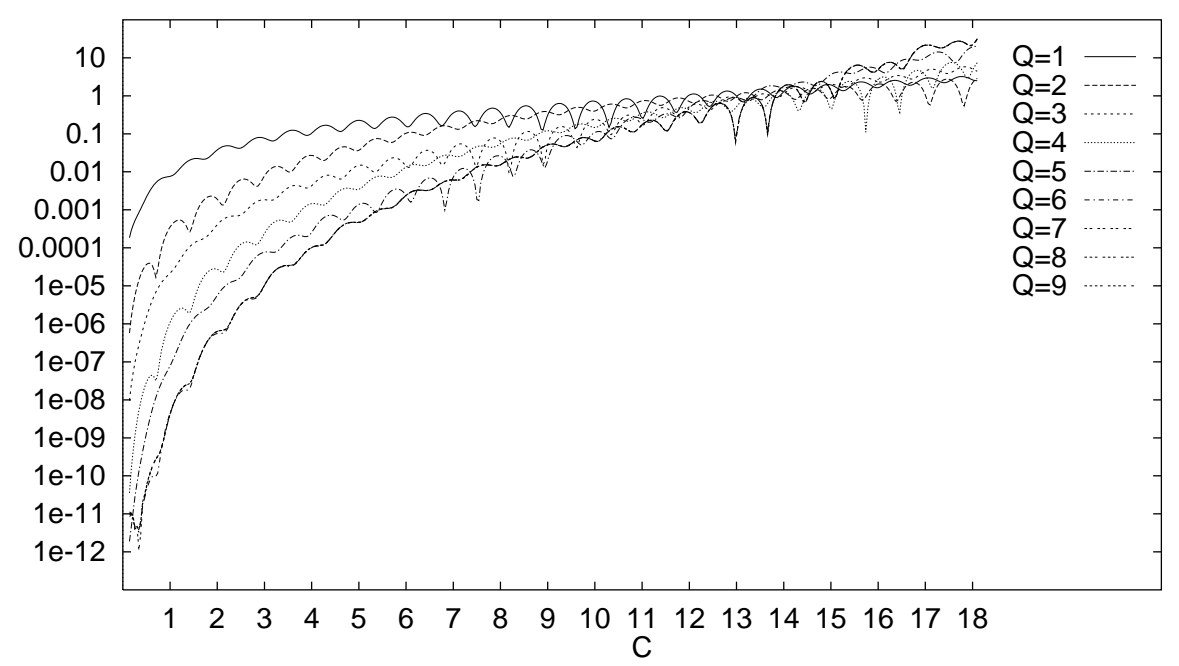

FiguRE 6. RMS error for the derivative of the function (78) with $G=48$ and amplitudes, including the principle amplitudes $A_{1}^{0}, A_{2}^{0}$, determined by a mixture of the available equations when $\gamma_{1}=$ $0.1, \gamma_{2}=4.55$.

\section{Discussion}

The basic idea utilized in this paper is to split a singular function into two parts, namely a relatively smooth part and a specially structured singular part. The idea is not new as references given earlier in this paper show (see also [4] and the references given there). As far as we know, however, a systematic and detailed analysis of such a splitting has not been carried out earlier. We have been able to do this analysis in a relatively general setting in such a way that a systematic analysis of each of the two parts has been possible. Furthermore, from the analysis we have been able to construct a new class of spectral methods based on the Fourier method. This new class of methods is much more flexible than the traditional spectral methods for instance in applications to differential and integral equations. Applications to partial differential equations in complex geometries have already been given in [11], [14], and further applications will be given elsewhere. The analysis also establishes relations between the continuous and the discrete Fourier coefficients which for instance may be useful in relating results obtained by the collocation method with results obtained by the Galerkin method.

A key problem in the new class of spectral methods for functions with singularities, is the determination of the singularity amplitudes. A large part of the present paper has therefore been devoted to that issue, and the numerical results show that quite reasonable accuracies can be obtained by the recommended algorithms. In fact, with approximately the same amount of work as in a traditional Chebyshev method, approximately the same accuracy and resolution properties [17] can be achieved by the new method with the added bonus of a uniform grid and the attractive flexibility with respect to the locations of the singularities, e.g. the boundary points. To be fair, it should here be added, however, that for functions which are very well resolved, the traditional Chebyshev method will give better 
accuracies than the new method since the latter is of finite order. We note that in the system of equations we have established for the singularity amplitudes, the coefficient matrix is uniquely determined by the locations of the singularities $\gamma_{j}$, $j=1, \ldots, M$, i.e. by the geometry of the problem.

Since the analysis shows that the order $Q$ in the representation (37) normally should be chosen as a relatively small number, the problem of determining when the singular part converges if $Q \rightarrow \infty$ is rather an academic problem. The answer to this academic problem is easily obtained, however, when $V_{n}(x, \gamma)=$ $U_{\alpha+n}(x-\gamma)$ or $V_{n}(x, \gamma)=U_{\alpha+n}(\gamma-x)$ for some given $\alpha$. In fact, the asymptotic result (31) shows that the functions $V_{n}(x, \gamma)$ then essentially group into only 4 distinct functions which actually are translates of each other. Thus a necessary and sufficient condition for absolute convergence is that for each $j=1, \ldots, M$ the infinite sums $\sum_{m=0}^{\infty} A_{j}^{n+4 m}$ converge for $n=0,1,2,3$. As examples, we therefore immediately see that if $w(x)=w_{c}(x)$ either is given by (78) or by

$$
w_{c}(x)= \begin{cases}0 & \text { when } x \in\left[0, \gamma_{1}\right), \\ e^{c x} & \text { when } x \in\left[\gamma_{1}, \gamma_{2}\right] \\ 0 & \text { when } x \in\left(\gamma_{2}, 2 \pi\right]\end{cases}
$$

where $c, \gamma_{1}, \gamma_{2}$ are constant parameters such that $0 \leq \gamma_{1}<\gamma_{2}<2 \pi$, then we will have convergence if and only if $|c|<1$. It thus seems that the analysis of convergence when $Q \rightarrow \infty$ is considerably simpler in our setting than in the setting given in [21].

The primary application area we have had in mind in this paper has been applications involving piecewise smooth functions where the special feature functions $V_{n}(x, \gamma)=U_{n}(x-\gamma)$ are given by (2) and the Bernoulli polynomials. As briefly indicated in section 6 , however, we hope to apply the more general family of special feature functions $U_{\alpha}$ for important multi-dimensional problems in the near future. Finally, we would like to add that it is not difficult to find both one- and multi-dimensional problems which may require other types of special feature functions than the family $U_{\alpha}$ described in this paper. The functions describing corner singularities [22] may here serve as illustrating examples.

\section{ACKNOWLEDGEMENT}

The author wishes to express his gratitude to Professor David Gottlieb and his group at the Division of Applied Mathematics, Brown University, Providence, R.I., U.S.A. for their generous hospitality and many fruitful discussions during the 3

different stays the author had there in 1994-96, when parts of this work was carried out.

\section{REFERENCES}

[1] T. M. Apostol, Calculus, Vol. II, Second Edition, Wiley, New York, NY, (1969). MR 40:1542

[2] E. Anderson, Z. Bai, C. Bischof, J. Demmel, J. Dongarra, J. Du Croz, A. Greenbaum, S. Hammarling, A. McKenney, S. Ostrouchov, and D. Sorensen, LAPACK Users' Guide, Society for Industrial and Applied Mathematics, Philadelphia, PA, (1992).

[3] C. M. Bender and S. A. Orszag, Advanced Mathematical Methods for Scientists and Engineers, McGraw-Hill, New York, NY, (1978). MR 80d:00030

[4] J. P. Boyd, Chebyshev \& Fourier Spectral Methods, Lecture Notes in Engineering 49, Springer-Verlag, Berlin, (1989).

[5] K. P. Bube, $C^{m}$ convergence of trifonometric interpolants. SIAM J. Numer. Anal. 15, (1978), pp. 1258-1268. MR 80g:42002 
[6] C. Canuto, M. Y. Hussaini, M. Yousuff, A. Quarteroni and T. A. Zang, Spectral Methods in Fluid Dynamics, Springer-Verlag, New York, NY, (1988). MR 89m:76004

[7] P. J. Davis and P. Rabinowitz, Methods of Numerical Integration, Second Edition, Academic Press, Orlando, FL, (1984). MR 86d:65004

[8] K. S. Eckhoff, Accurate and efficient reconstruction of discontinuous functions from truncated series expansions. Math. Comp. 61, (1993), pp. 745-763. MR 94a:65073

[9] K. S. Eckhoff, On discontinuous solutions of hyperbolic equations. Comput. Methods Appl. Mech. Engrg. 116, (1994), pp.103-112. MR 95c:65163

[10] K. S. Eckhoff, Accurate reconstructions of functions of finite regularity from truncated Fourier series expansions. Math. Comp. 64, (1995), pp. 671-690. MR 95f:65234

[11] K. S. Eckhoff, On a high order numerical method for solving partial differential equations in complex geometries. J. Scient. Comp. 12 (1997), pp. 119-138.

[12] K. S. Eckhoff and J. H. Rolfsnes, A Fourier method for nonsmooth hyperbolic problems. Proc. 3. Internat. Conf. Spectral and High Order Methods, ICOSAHOM'95 (Houston, Texas, U.S.A., 1995), edited by A.V. Ilin and L.R. Scott (Houston Journal of Mathematics, 1996), pp. 109-119.

[13] K. S. Eckhoff and J. H. Rolfsnes, On nonsmooth solutions of linear hyperbolic systems. J. Comp. Phys. 125, (1996), pp. 1-15. MR 96k:65067

[14] K. S. Eckhoff and C. E. Wasberg, Solution of parabolic partial differential equations in complex geometries by a modified Fourier collocation method. Proc. 3. Internat. Conf. Spectral and High Order Methods, ICOSAHOM'95 (Houston, Texas, U.S.A., 1995), edited by A.V. Ilin and L.R. Scott (Houston Journal of Mathematics, 1996), pp. 83-91.

[15] A. Erdélyi, W. Magnus, F. Oberhettinger and F. C. Tricomi, Higher Transcendental Functions, McGraw-Hill, New York, NY, (1953). MR 15:419i

[16] G. H. Golub and C. F. Van Loan, Matrix Computations, Second Edition, John Hopkins University Press, Baltimore, MD, (1989). MR 90d:65055

[17] D. Gottlieb and S. A. Orszag, Numerical Analysis of Spectral Methods: Theory and Applications, CBMS-NSF Regional Conference Series in Applied Mathematics 26, Society for Industrial and Applied Mathematics, Philadelphia, PA, (1977). MR 58:24983

[18] L. V. Kantorovich and V. I. Krylov, Approximate Methods of Higher Analysis, P. Noordhoff Ltd, Groningen, (1958). MR 21:5268

[19] H.-O. Kreiss and J. Oliger, Stability of the Fourier method. SIAM J. Numer. Anal. 16, (1979), pp. 421-433. MR 80i:65130

[20] C. Lanczos, Discourse on Fourier Series, Oliver \& Boyd, Edinburgh, (1966). MR 33:7772

[21] J. N. Lyness, Computational techniques based on the Lanczos representation. Math. Comp. 28, (1974), pp. 81-123. MR 48:12777

[22] G. Strang and G. J. Fix, An Analysis of the Finite Element Method, Prentice-Hall, Englewood Cliffs, NJ, (1973). MR 56:1747

[23] H. Vandeven, Family of spectral filters for discontinuous problems. J. Scient. Comp. 6, (1991), pp. 159-192. MR 92k:65006

[24] A. Zygmund, Trigonometric Series, Vol I, Cambridge University Press, Cambridge, (1968). MR 38: 4882

Department of Mathematics, University of Bergen, Johannes Bruns gate 12, N-5008 Bergen, Norway

E-mail address: reske@mi.uib.no 